\title{
Free Electron Laser
}

\author{
Pascal Elleaume \\ CEN/Saclay, Gif-sur-Yvette and LURE, Orsay
}

\section{and Yves Farge}

LURE, Orsay and the Ministère de la Recherche et de la Technologie

The generation of radiation by electric charges is a phenomenon that has been well known since the last century. It can arise from changes in level of bound electrons when the radiation is then essentially monochromatic with a frequency related to the transition energy of the bound electrons, or from the acceleration of free or almost free electrons, as for example in classical antennas, klystrons or black body radiation and synchrotron radiation. Lasers have been known since the turn of the fifties where bound electrons in atoms, molecules or crystals are the source of oscillation and mirrors provide feedback to give the amplification.

More recently, another way of producing a light amplifier was discovered ${ }^{1}$ ), making use of free relativistic electrons travelling in a transverse periodic magnetic field called an undulator. Lasers built on these principles are called Free Electron Lasers (FEL).

In the following, we focus on the Compton type FEL where electrons individually experience the undulator field and the photon field (and we neglect repulsive electrostatic interactions between the electrons). There also exist high electron density FEL where electrons collectively interact with the undulator and photon fields.

\section{Resonance Between the Radiation} Field and Electrons

Two kinds of undulator have been made so far: the planar as first developed by Motz ${ }^{2}$ ) at the beginning of the fifties which comprises a series of magnets of alternating field, and the helical as made by $\mathrm{J}$. Madey and his coworkers ${ }^{1}$ ). In a planar undulator, the electrons propagate along the $z$-axis and the magnetic field has one component only oscillating along the $z$-axis:

$$
\text { i.e. } \quad \vec{B}=B_{0} \vec{u}_{x} \sin \left(2 \pi z / \lambda_{0}\right)
$$

where $\vec{u}_{\mathrm{x}}$ is a unit vector parallel to the $x$-axis and $\lambda_{0}$ is the undulator spatial period.

In a helical undulator, the field is still transverse but turns around the $\mathrm{z}$-axis like a helix:

$$
\text { i.e. } \begin{aligned}
\vec{B} & =B_{0} \vec{u}_{x} \sin \left(2 \pi z / \lambda_{\mathrm{o}}\right) \\
& +B_{\mathrm{o}} \vec{u}_{y} \cos \left(2 \pi z / \lambda_{\mathrm{o}}\right)
\end{aligned}
$$

where $\vec{u}_{y}$ is a unit vector parallel to the $y$ -axis. The physics of the interaction of the electron with the undulator field leading to the FEL is essentially the same for both kinds of undulator and for greater simplicity we shall discuss the planar.

The undulator forces the electrons to follow a sinusoidal trajectory (Fig. 1) but for relativistic electrons having a total energy: $\xi=\gamma m_{0} c^{2}$ where $\gamma \gg 1$ and $m_{0} c^{2}$ is the rest mass energy $(=0.511 \mathrm{MeV})$, the amplitude of the oscillatory motion in the undulators would normally be very small $(\sim \mathrm{mm})$ compared to the undulator period $(\sim 10 \mathrm{~cm})$.

If, however we assume that an electromagnetic plane wave propagates in the same direction as the electron, the wave and the electrons will be in resonance when $\lambda$ (the wave period), $\lambda$ (the undulator period) and $v_{z}$ (the electron longitudinal velocity) are as illustrated in Fig. 1: during the time a given electron moves through one period of the magnet, the plane wave advances one optical period with respect to the electron. This resonance is behind the special features of the emission of light by the electrons in the undulator (spontaneous emission) and of the amplification leading to the FEL.

In Fig. 1, at time $t_{0}$, the electron coincides with a period of the wave indicated by the black mark. The plane wave is travelling faster than the electron and at time $t$ and position $z$, the phase of the plane wave is given by $\phi=\omega t-k z$ where $\omega=2 \pi c / \lambda$ and $k=2 \pi / \lambda$.

Again referring to Fig. 1, resonance occurs when the plane wave has travelled a distance $\lambda_{0}+\lambda$ during a time $\lambda_{0} / v_{2}$ which can be expressed by the equation:

$$
\begin{aligned}
0 & =\Phi\left(t_{0}+\lambda_{0} / v_{2}\right)-\Phi\left(t_{0}\right) \\
& =\omega \lambda_{0} / v_{z}-k\left(\lambda_{0}+\lambda\right)
\end{aligned}
$$

This relation can be rewritten as:

$$
\lambda=\lambda_{0}\left[\left(c / v_{2}-1\right)\right]
$$

For technical reasons, a typical undulator period cannot be shorter than several centimetres while Eq. 1 shows that $c / v_{z}$ must be close to unity to achieve resonance with electromagnetic waves in the visible or the UV. Electrons must therefore be relativistic and the higher their energy, the shorter will be the resonant wavelength.

From the relation

$$
\gamma^{-1}=\sqrt{ }\left[1+\left(v_{z}^{2} / c^{2}\right)+\left(v_{\perp}^{2} / c^{2}\right)\right]
$$

where $v_{1}$ is the transverse velocity due to the undulator magnetic field (which can be calculated using the Lorentz equations), it is possible to rewrite Eq. 1 as:

$$
\lambda=\lambda_{0}\left(1+1 / 2 K^{2}+\gamma^{2} \vartheta^{2}\right) / 2 \gamma^{2}
$$

where $K=93.4 B_{0} \lambda_{0}$ (MKS units)

$B$ is the undulator peak magnetic field

$\vartheta^{\circ}$ is the angle between the electron trajectory and the propagation axis of the plane wave.

\section{Gauge Theories in Particle Physics}

\section{J R Aitchison \& A J G Hey}

A new, intuitive approach to the ideas and methods of gauge field theories in elementary particle physics,

presenting this advanced theoretical topic at a level which is accessible to experimental physicists and new graduate students, and concentrating on the need of experimentalists for a lower-level approach than in previous publications.

1982 xiv $+332 p p$

0-85274-534-6 £14.50

\section{A European Experiment}

\section{The Launching of the JET} Project

\section{Denis Willson}

A revealing account of how national and supranational politics affected the building of a major fusion energy experiment, the Joint European Torus, within the European

Community. Writing from personal experience, the author explains how national interests prevailed over scientific expediency and reveals how the scientists inevitably became involved in the political struggles that attracted much public attention and delayed construction for two years.

$1981 \mathrm{x}+178 \mathrm{pp}$

hardcover $0-85274-543-5 \quad £ 10.50$

flexicover $\quad 0-85274-549-4 \quad € 6.95$

\section{Parallel \\ Computers}

Architecture, Programming and Algorithms

R W Hockney \& C R Jesshope A practical and comprehensive introduction to the architecture and associated software of operating supercomputers, combining an advanced text for students with a reference for professional users. It describes and compares several successful machines and discusses the choice of numerical algorithms and suitable programming languages for specific machines or applications. 1981 xii $+416 p p$

$0-85274-422-6 \quad$ f22.50

Adam Hilger Ltd Techno House Redcliffe Way Bristol BS1 6NX England 
Undulator

magnetic field

$\odot$

$\odot$

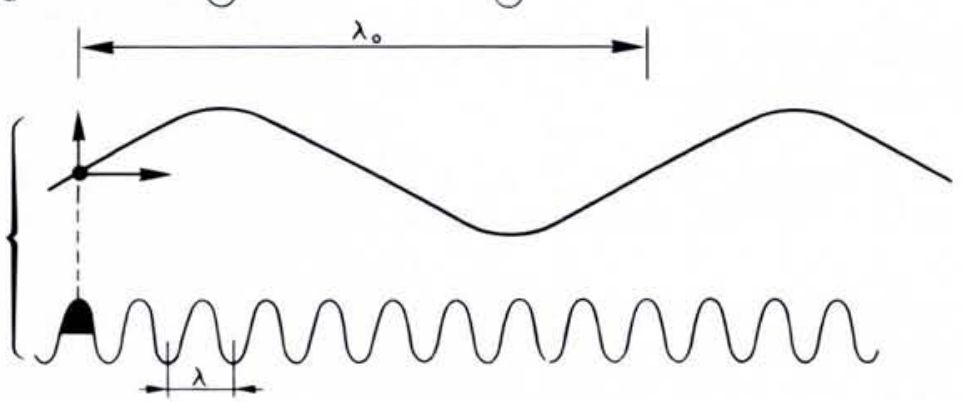

at time
$t_{0}+\frac{\lambda_{0}}{2 v_{z}}$

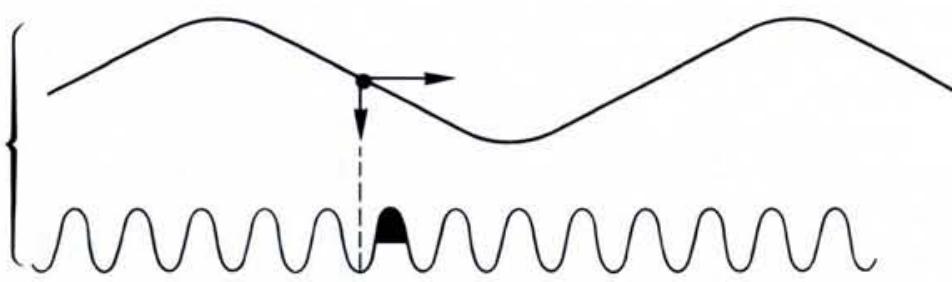

at time
$t_{0}+\frac{\lambda_{0}}{V_{z}}$

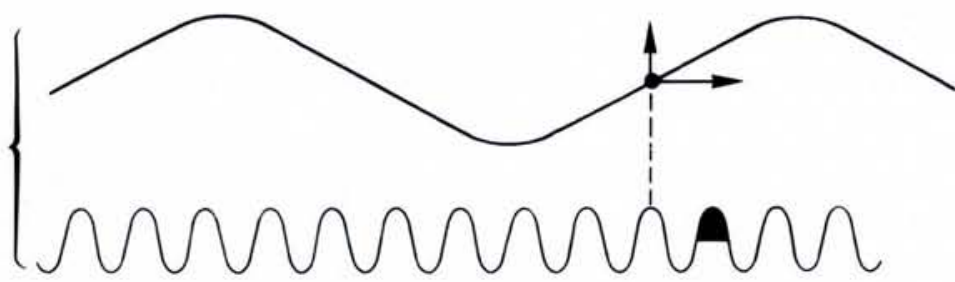

Fig. 1 - Electron and light dynamics in an undulator: at resonance the electron is overtaken by one period of the photon field as it passes through one period of the undulator.

So far we have only considered first order resonance, but there can be higher order resonance if the electron is passed by an integer number of periods of the plane wave. Using the same arguments as for the first order, it is readily shown that the wavelength for the $n^{\text {th }}$ order resonance is the wavelength for the first order (given by Eq. 2) divided by $n$.

\section{Spontaneous Emission}

Relativistic electrons radiate light when they traverse a magnetic field - the wellknown synchrotron radiation which is now a very important tool in the UV and X-ray spectral range. This emission has a very broad and continuous spectrum, but let us consider the part of this spectrum with a wavelength $\lambda$ close to the resonant condition defined in Fig. 1 and in Eq. 2. It is seen from Fig. 1 that at any given time, the synchrotron radiation at wavelength $\lambda$ emitted by the electron will be in phase with that emitted by the same electron one period before. The conclusion is that, at the wavelength given by Eq. 2, the synchrotron radiation emitted by an electron over each of the $N$ periods of the undulator will interfere positively, and the power emitted around this wavelength will be $N^{2}$ times the power emitted from one period alone. At lower or higher wavelengths than $\lambda$, destructive interference occurs giving a rela- tive spectral width of the peak radiation equal to $1 / N$.

\section{Amplification of an Electromagnetic Plane Wave}

In this section, we shall see how a plane wave close to resonance with the electrons can be amplified. From the Lorentz force it is known that:

$$
\Delta \xi=-\int e \vec{v} \vec{E} d t
$$

where $e$ is the electron charge

$\vec{E}$ is the plane wave electric field $v$ is the electron velocity

$\Delta \xi$ is the electron energy change in the undulator.

Usually $\vec{E}$ is oscillating so fast that the integral (3) averages out to zero. However at resonance, remembering that $E_{z}=0$, we see from Fig. 1 that $\vec{v} \vec{E}$ retains the same sign at $t_{0}, t_{0}+c / 2 v_{2}, t_{0}+c_{0} / v_{z}$ indeed at all times. $\triangle \xi$ can be either positive or negative so that some electrons are decelerated, others are accelerated when they come close to resonance with the incident plane wave. Simply from inspecting Fig. 1 we see that if an electron is strongly decelerated, another electron located $\lambda / 2$ ahead undergoes a strong acceleration. As a typical electron bunch length is much larger than the optical wavelength, it might be expected that the total energy change over all electrons would be zero. However it can be shown from the exact solution of the
Lorentz equations ${ }^{4}$ ) that when electrons are slightly shifted from resonance with respect to the plane wave, a positive or negative total energy balance can result. This occurs to a second order in the interaction. It can also be shown that the energy gained or lost by the electrons is taken from or given to the incident wave which is consequently absorbed or amplified.

Fig. 2 plots the gain and spontaneous emission curves obtained at Orsay ${ }^{3}$ ), as a function of electron energy at wavelength $\lambda=0.488 \mu \mathrm{m}$. The negative part of the gain curve corresponds to absorption, whereas zero gain coincides with the maximum for spontaneous emission at the exact energy for resonance. The spontaneous emission curve appears to be the derivative of the gain curve as predicted from theory, and the relative width of both curves is closely related to the undulator perind number.

Colson has given an expression for the maximum gain for an $N$ period helical undulator:

$G_{\max }=0.27 e^{4} B^{2} \rho_{\mathrm{e}} \lambda_{\mathrm{o}}\left(N \lambda_{\mathrm{o}} / \gamma m_{\mathrm{o}} c^{2}\right)^{3}$ (4) where $\rho_{\mathrm{e}}$ is the electron density and $B_{\mathrm{o}}$ is the undulator magnetic field.

Just as amplification by the stimulated emission process is responsible for the laser effects in atoms, molecules and crystals, amplification by this resonant interaction between light and free electrons (in the sense that they are not bound to any nucleus) leads to the Free Electron Laser process. Moreover, the coherence of the FEL will be just the same as with a regular laser since it too has its root in the amplification process ${ }^{5}$ ).

Let us note that we have discussed the FEL completely in classical terms, and furthermore that all the experimental data obtained so far can be classically explained. This fact may intrigue a reader used to the idea that lasers are typical quantum devices, but this is true only for regular lasers where the bound electrons emitting the light can be properly described by quantum mechanics alone. A quantum description of the concept of resonance is given by the inverse resonant Compton scattering process first proposed by Dirac and Kapitza ${ }^{6}$ ); the amplification results from stimulated Compton scattering. The quantum mechanical description was the first theory of the FEL developed by J.M.J. Madey ${ }^{7}$ ) who had enough confidence in his predictions to build six years later the first FEL ${ }^{8}$ ).

As in regular lasers, there will be a saturation mechanism limiting the power output. Energy loss from an electron in the undulator increases with the light power, and at high power, this shift is such that Eq. 2 is only true in a part of the undulator. The electrons then can provide amplification in some part of the undulator and absorption or zero gain in other parts; this is the onset of saturation. 


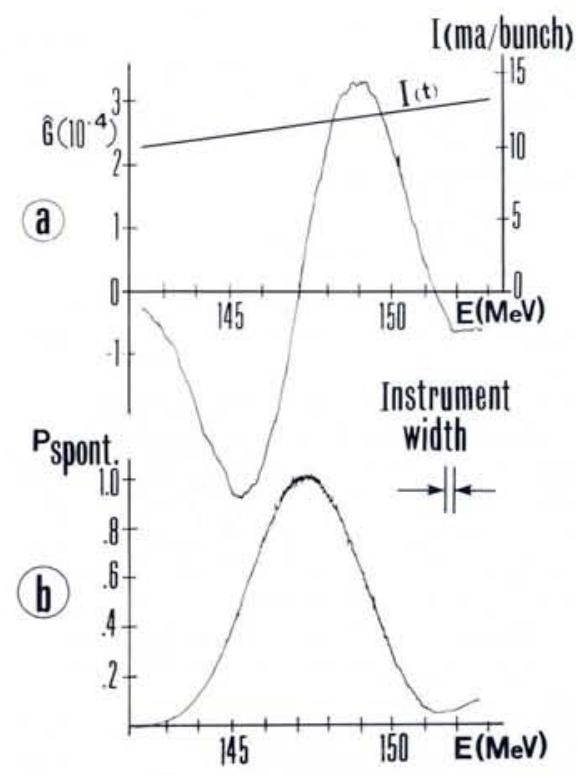

Fig. 2 - a) Experimental gain for a given wavelength versus electron energy of the superconducting planar sinusoidal undulator on the $A C O$ storage ring.

b) Spontaneous emission at the same wavelength versus electron energy.

Both curves are in good agreement with theory which predicts that the gain must be proportional to the derivative of the spontaneous emission.

Typical energy shift for saturation is $\Delta \gamma / \gamma \cong \mathrm{I} / N$, i.e. as we have seen earlier, the same order as the relative width of spontaneous emission and therefore of gain curves.

One conclusion is that the saturated power decreases when $N$ increases. However, since the maximum gain (Eq. 4) grows as $N^{3}$, there will be a trade off between maximizing the gain to be far enough above threshold, and maximizing the output power.

\section{First Experimental Results}

The first experiment in which gain was first observed was performed by Madey's group 1) at Stanford using a helical superconducting undulator and a small linear superconducting linac which was delivering high density electron bunches. The experiment had the following parameters:

$B_{0}=0.24 \mathrm{~T} \quad \gamma m c^{2}=24 \mathrm{MeV}$

$\lambda_{\mathrm{o}}=3.2 \mathrm{~cm} \quad \rho_{\mathrm{e}}=6.4 \times 10^{7} \mathrm{~cm}^{-3}$

$N=160$

The group used a $\mathrm{CO}_{2}$ laser to measure gain versus $\lambda$ (see Fig. 3 ) and obtained a maximum figure of $7 \%$ which is in fair agreement with the $4 \%$ expected theoretically. A similar result has been obtained recently in the visible spectrum with the $\mathrm{ACO}$ undulator at Orsay using the green or the blue line of an argon laser ${ }^{3}$ ) (see Fig. 2). After their gain measurement, the Stanford group constructed an optical cavity 12 $\mathrm{m}$ long. Operating with $43 \mathrm{MeV}$ electrons, laser operation was observed at $\lambda=3.4$ $\mu \mathrm{m}$ with an average power of $0.36 \mathrm{~W}$ and a peak power of $7 \mathrm{~kW}^{8}$ ). In this pioneering experiment, the Stanford group thus de- monstrated the feasibility of a free electron laser.

\section{FEL in the Infrared}

If we postulate now as the parameters: of an FEL structure -

$$
\begin{array}{ll}
\lambda_{\circ} & \sim 5 \mathrm{~cm} \\
K & \sim 2
\end{array}
$$$$
\text { and } \quad \gamma m c^{2} \sim 5-50 \mathrm{MeV}
$$

Eq. 2 gives $\lambda \sim 800-8 \mu \mathrm{m}$, which in dicates that we should have an FEL operating in the infrared. Such an electron energy range is available from linacs (experiments at Stanford, Los Alamos, MSNW and TRW), microtrons (Frascati) or Van de Graaf (Santa Barbara).

As we have seen, saturation of the gain occurs in a regular undulator because at high power the extracted energy from the electrons is such that they cannot stay on resonance all along the undulator. The saturation limit can be pushed higher by compensating for the electron energy change, and progressively decreasing the period of the undulator along its length, so maintaining the electron in resonance with the plane wave. Such undulators are called tapered undulators and are presently being tested at Los Alamos, MSNW and TRW.

Computer calculations ${ }^{9}$ ) have shown that with a tapered undulator, it should be possible to convert up to 20 to $30 \%$ of the kinetic electron energy into the laser beam, reaching the terawatt range with modern electron pulsed accelerators. Such FELs are expected to be useful for plasma heating in fusion tokamaks. In addition, with many existing electron accelerators, it should be possible to build simple tunable lasers in the far infrared with undulators made of permanent magnets (samarium cobalt); such devices could be extremely useful in a wavelength range where tunable powerful sources do not exist.

FEL in the Visible and Ultraviolet

In storage rings, high density electron beams can circulate for periods of up to several days. Originally developed by high energy physicists for studying electronpositron collisions, new machines are now under construction all over the world dedicated to the production of synchrotron radiation. These machines are optimized to be photon sources as bright as possible which means with the largest possible electron density. On a straight section of such a storage ring, it is possible to insert an undulator (Fig. 4). Two experiments of this kind are under way, one on the VEPP-3 ring at Novosibirsk, the second on the ACO ring at Orsay. Two others are in preparation at Frascati and Brookhaven respectively.

The description of the gain mechanism has been given for a sinusoidal undulator, but another magnetic configuration has been proposed by Vinokurov and Skrinsky ${ }^{10)}$ which gives a larger gain for the same length, and is called the "optical klystron". It is made of two identical undulators separated by a space in which a magnetic field induces a single large wiggle in the electron trajectory. Optical klystrons are under test at Novosibirsk and Orsay. Typical energies for these rings are $150-800$ $\mathrm{MeV}$ which allows resonance and therefore FEL operation in the visible and ultraviolet.

If one is confident in the predictions, one can expect that new storage rings under construction will give lasers that are tunable from $0.2 \mu \mathrm{m}$ to about $20 \mu \mathrm{m}$ with a

Fig. 3 - Schematic description of the Stanford experiments on a helical undulator with a $3.2 \mathrm{~cm}$ period (taken from Refs. 1 and 8).

a) The electron beam is travelling through the helical undulator with a $\mathrm{CO}_{2}$ laser beam. The gain is measured by varying the electron energy.

b) A $12 \mathrm{~m}$ long optical cavity is added to the undulator to make a laser; its length is calculated to fit the distance separating electron bunches.

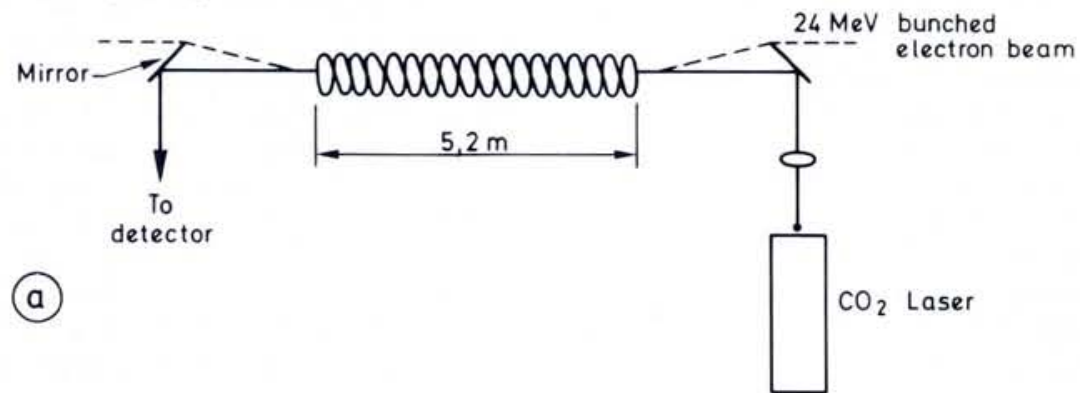

(b)

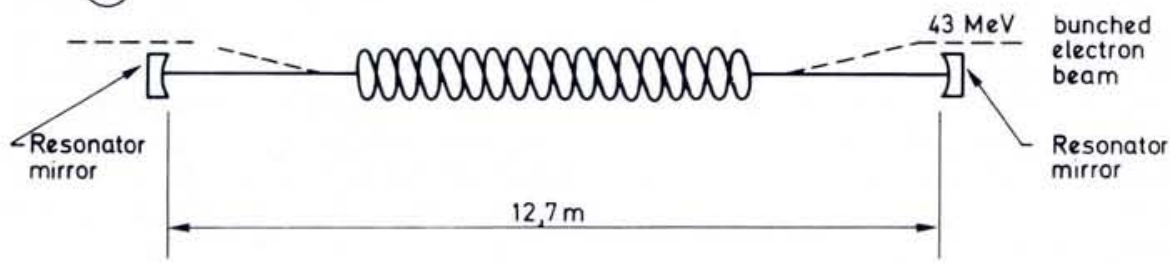




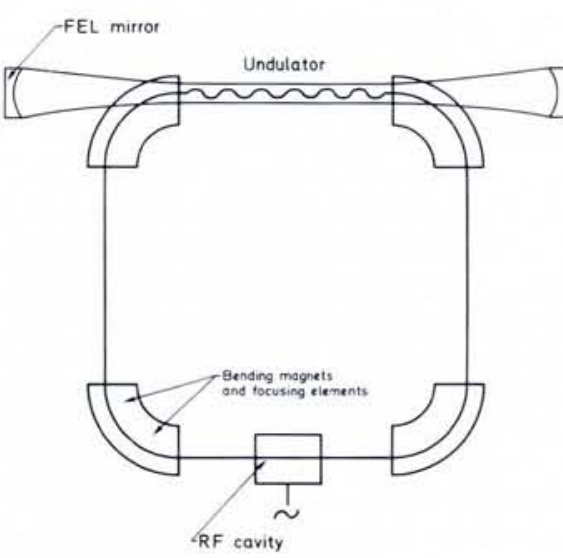

Fig. 4 - Schematic drawing of an FEL on a straight section of a storage ring.

$\mathrm{CW}$ power between 10 and $100 \mathrm{~W}$ and pulse lengths of about $10-30$ ps separated by about $100 \mathrm{~ns}$. Such lasers would be extremely useful especially in photo-chemistry. Nevertheless, even if recent experiments have shown good agreement with theory, the feasibility of such devices has still to be proved.

\section{Conclusions}

We have briefly summarized the work under way in the field of the Compton type free electron lasers. It is very interesting to note that acceleration of relativistic electrons produces the same phenomena as electronic transitions in atoms, molecules or solids: spontaneous emission and gain. It is also important to note that while FEL theory is quite simple, its development as well as experimental verification have been delayed by a traditional lack of interaction between machine physicists (who were working only on particle physics accelerators) and laser physicists who were busy with their solid or gas amplifiers.

It is quite realistic to expect the field to develop very quickly during the eighties and to foresee FELs working from the millimeter range to the vacuum UV before 1990, with pulsed and electrostatic accelerators in the far infrared and infrared, and with storage rings in the IR, visible and UV.

The possibility of reaching the soft X-ray or $\mathrm{X}$-ray range with an FEL is a common question. We do not believe in such a possibility because the gain drops too quickly with the frequency and optical mirrors become very poor beyond $0.16 \mu \mathrm{m}$. With storage rings, one obtains the highest electron density feasible today (higher densities with such machines cannot be reached because of the repulsive interaction bet- ween electrons in the bunches) and no one has yet suggested a way to build any other kind of accelerator which could give energies of the order of $1 \mathrm{GeV}$ with much higher electron densities.

\section{REFERENCES}

1. Elias L.R., Fairbank W.M., Madey J.M.J., Schwettman M.A. and Smith T.I., Phys. Rev. Lett. 36 (1976) 717.

2. Motz H., J. Appl. Phys. 22 (1951) 527.

3. Bazin C., Billardon M., Deacon D.A.G., Elleaume P., Farge Y., Madey J.M.J., Ortega J.M., Petroff, Y., Robinson K.E. and Velghe M., O.N.R. Workshop on FEL (Sun Valley), to be published in Physics of Quantum Electronics, 8-9 (Addison-Wesley) 1982.

4. Colson W.B., Phys. Letters A 59 (1976) 187. 5. Kastler A., La Recherche 128 (1981) December.

6. Kapitza P.L. and Dirac P.A.M., Proc. Cambridge Phys. Soc. 29 (1933) 297.

7. Madey J.M.J., J. Appl. Phys. 42 (1971) 1906. 8. Deacon D.A.G., Elias L.R., Madey J.M.J., Ramian G.J., Schwettman H.A. and Smith T.I., Phys. Rev. Lett, 38 (1977) 892.

9. Kroll N.M., Morton P. and Rosenbluth N.M., Physics of Quantum Electronics, 7 (Addison Wesley) 1982, eds. S.F. Jacobs, H.S. Pilloff, M. Sargent III, M.O. Scully, R. Spitzer.

10. Vinokurov N.A. and Skrinsky A.N., Proc. 10th Int. Conf. on High Energy Charged Particle Accelerators, Serpukhov, Vol. 2 (1977), p. 454.

\section{Research on the Structure of Matter in Italy Carlo Rizzuto, Rome (Chairman GNSM)}

20 years Development and the National Group for the Structure of Matter (GNSM) - an overview of basic and applied research on the structure of matter in Italy (covering condensed matter, atomic and molecular physics) and how it has been developed by the GNSM.

The system of organizing research through national groups adopted by the National Research Council (CNR) has been very successful in various fields of physics (and engineering, see panel 1) and for Italy's Finalized Programmes on applied research. (It could even be applied on an international basis in specific circumstances.) The GNSM Group was founded informally in 1962 when most of Italy's research in the field came under the National Research Council after an initial period of funding, through the late $50 \mathrm{~s}$, by the National Institute for Nuclear Physics (INFN). Official acknowledgement dates back to 1967, when it was recognized as a fully titled organisation with a Statute and a Scientific Council.

The Group now consists of 34 Research Units and 5 Laboratories. Research Units including technical and administrative staff range in size from a minimum of five to a maximum of 58 total staff and they operate inside universities and certain other institutions. A single scientific supervisor is responsible but the research covers several (two to six) different fields. The Laboratories which also cover different fields employ between 20 and 35 staff members and are headed by a Director of Research.

The Group's Scientific Council is made up of the Supervisors of the Units and the Directors of the Laboratories together with six elected representatives of the research and technical staff and five coopted scientific experts from industry or research institutions. The executives of the Group are the Chairman of the Scientific Council, and a Board of 10 members elected by the Council. A central office in Rome with a staff of five people is the operating base and is directed by the Group's Director.

\section{Resources}

The basic budget, allocated in about equal proportions by the CNR and the Ministry of Public Instruction (see Panel 2) for research expenses of GNSM in 1981 (excluding salaries but including overheads) was approximately $4.85 \times 10^{6}$ US\$ of which roughy $3 \times 10^{6}$ US\$ went to the Research Units, and $1.25 \times 10^{6}$ US\$ to the
Panel 1

Non-nuclear physics in Italy is supported through five research groups under the CNR. GIFCO (Italian Group for Cosmic Physics) 4 Labs, 2 Res. Units;

GNA (National Group for Astronomy) 2 Labs., 5 Res. Units:

GNCB (National Group for Cybernetic and Biophysics) 3 Labs., 13 Res. Units and Centres:

GNEQP (Nat. Group for Quantum Electronics and Plasms) 2 Labs., 12 Res. Units and Centres:

GNSM (Nat. Group for Structure of Matter) 5 Labs, 34 Res. Units:

Nuclear Physics is supported by an independent institution, the National Institute for Nuclear Physics (INFN)

\section{Panel 2}

Starting from 1980, university research has been funded on a regular basis also from the Min. of Public Instruction. The Overall fun ding for all non-nuclear physics in university and CNR was, for 1981, approximately $3.75 \times$ $10^{6}$ US\$ from MPI and $12.25 \times 10^{6}$ US\$ from CNR, the allocation of the funds to the various subfields of physics being made by two con sulting committees (one for each) elected by all physicists. Nuclear physics is funded directly by the government and received in 1981, in cluding the international programmes, approximately $75 \times 10^{6}$ US $\$$ of which approximately $33.3 \times 10^{6}$ US\$ was for CERN. 